SHOULD WE GIVE UP DOMAIN IMPORTANCE WEIGHTING IN QOL MEASURES?

Chang-ming Hsieh 


\title{
SHOULD WE GIVE UP DOMAIN IMPORTANCE WEIGHTING IN QOL MEASURES?
}

\author{
Abstract \\ The purpose of this article is to examine the recent claims calling for abolishing domain \\ importance weighting in quality of life (QoL) measures by considering the evidence conceptually \\ and empirically. Based on a close review of evidence presented to date, it is suggested that using \\ the range-of-affect hypothesis as a possible explanation of the poor performance of weighted \\ satisfaction composite in predicting or correlating with global satisfaction or QoL measures can \\ be beneficial to our understanding of the life satisfaction literature. However, given the \\ conceptual focus and the empirical approach of the range-of-affect hypothesis presented in the \\ life satisfaction context, using the range-of-affect hypothesis to argue against domain importance \\ weighting raised more questions than answers. Calling for abolishing domain importance \\ weighting in QoL measures, based on the evidence of range-of-affect hypothesis, is premature.
}


SHOULD WE GIVE UP DOMAIN IMPORTANCE WEIGHTING IN QOL MEASURES?

Introduction

Ten years ago, Trauer and Mackinnon (2001) examined the practice of using multiplicative scores (multiplying satisfaction and importance ratings) to measure quality of life (QoL). After discussing a number of conceptual, psychometric and empirical issues regarding multiplicative scores, Trauer and Mackinnon (2001) called for abandoning the use of multiplicative scores. However, as clearly stated by Trauer and Mackinnon (2001), they did not mean that all the domains of life should be of equal importance to the individual, rather it was the use of multiplicative scores that they were against. Since then, numerous studies have investigated the role of domain importance in QoL measures (e.g., Hsieh, 2003, 2004; Rojas, 2006; Russell \& Hubley, 2005; Russell, Hubley, Palepu, \& Zumbo, 2006). Although the results on the role of domain importance weighting in QoL measures remained mixed and by no means conclusive, recently, there have been repeated calls for abolishing not only multiplicative scores but also domain importance weighting entirely (e.g., Wu, 2008a, 2008b; Wu \& Yao, 2006a, 2006b, 2007). It should be noted that domain importance weighting is generally accomplished in one of the following three ways. One approach is to assign externally determined weights to various domains on a theoretical or conceptual basis. Although these weights reflect the concept of importance, the weights are applied to all respondents without taking into account any potential individual differences. A second approach is to consider regression coefficients as weights in a regression analysis when individual domain satisfaction scores are the predictor variables and a global QoL measure is the criterion variable. The results of this approach are heavily dependent on the criterion variable chosen and also do not take into consideration 
individual respondent differences. A third approach is to apply different domain importance weights to different respondents. This approach of domain importance weighting takes into account the potential individual differences in perceived importance of various domains and is the focus of this article.

As Russell and Hubley (2005) indicated, the debate over the role of domain importance weighting was not new and certainly was not unique to the area of QoL studies. Comparing the performances of weighted and unweighted scores has continuously produced mixed results (e.g., Russell \& Hubley, 2005; Russell et al., 2006; Skalli, Theodossioub \& Vasileioua, 2008). However, in light of the recent calls for abandoning domain importance weighting in QoL measures, one could not help but ask: Do we have enough evidence to give up domain importance weighting? The purpose of this article is to examine the claims calling for abolishing domain importance weighting in QoL measures by considering the evidence conceptually and empirically.

\section{Conceptual Ground}

The conceptual ground for much of the recent argument against domain importance weighting (see Wu, 2008a, 2008b; Wu \& Yao, 2006a, 2006b, 2007) was based on an extension of the literature on job satisfaction, specifically Locke's "range-of-affect" hypothesis (Locke, 1969, 1976, 1984). The range-of-affect hypothesis proposes that satisfaction with specific job facets (or domains) is determined on two subjective factors: 1) the perceived discrepancy between the amount of specific job facets that individuals feel they experience (have) and the amount they want to experience (or the "have-want discrepancy"), and 2) the importance that individuals perceive with various job facets. Based on the range-of-affect hypothesis, satisfaction with a particular job facet is a function of the have-want discrepancy for the job facet and the 
perceived importance of that job facet (Locke, 1969, 1976). Facet or domain satisfaction is, therefore, influenced by the interaction of the domain have-want discrepancy and domain importance. Especially, at a given level of discrepancy, a domain with high importance can produce a wide range of satisfaction level, while a domain with low importance can only produce a restricted range of satisfaction level -- around the center of the satisfaction-dissatisfaction spectrum. Evidence supporting the range-of-affection hypothesis has mostly appeared in job satisfaction literature (e.g., McFarlin, Coster, Rice \& Coopper-Alison, 1995; McFarlin \& Rice, 1992; Rice, Gentile \& McFarlin, 1991; Rice, Markus, Moyer \& McFarlin, 1991), and more recently, in life satisfaction literature (e.g., Wu, 2008a, 2008b; Wu \& Yao, 2006a, 2006b, 2007).

Support for the range-of-affect hypothesis is typically demonstrated by a significant discrepancy by importance interaction effect in a regression/ANOVA type of analysis with domain satisfaction as dependent variable. In job satisfaction literature, researchers have demonstrated that the relationship between discrepancy amount and satisfaction was stronger in high importance conditions than in the low importance conditions (e.g., Rice, Gentile et al., 1991) and the relationship between the amount of domain discrepancy and domain satisfaction was generally stronger for domains with higher importance than domains with lower importance (e.g., Rice, Markus et al., 1991). Extending the view of the range-of-affect hypothesis, Locke $(1969,1976)$ argued that the relationship between domain satisfaction and overall job satisfaction should be an unweighted additive one. That is, overall job satisfaction should be a sum of satisfactions across various domains of job without the need of weighting domain importance. The position was a natural extension of the range-of-affect hypothesis, as the hypothesis argued that domain satisfaction had already incorporated the judgment of domain 
importance, hence the concept of implicit weighting, according to Locke $(1969,1976)$.

Weighting domain satisfaction with domain importance would, therefore, be redundant. Based on the results that individual domain importance did not moderate the relationship between individual domain satisfaction and overall job satisfaction within most of the domains, researchers like McFarlin and Rice (1992) and McFarlin et al. (1995) suggested that the concept of implicit weighting was supported.

Recently, researchers (Wu, 2008a, 2008b; Wu \& Yao, 2006a, 2006b, 2007) applied the range-of-affect hypothesis in the life satisfaction context. Adapting the 12 facets/domains from the World Health Organization Quality of Life questionnaire (WHOQOL-100), Wu and Yao (2006b) found that in a sample of undergraduate students, individual domain satisfaction was significantly associated with the interaction of domain (have-want) discrepancy and domain importance. Wu and Yao (2006b) also found that domain importance did not moderate the relationship between individual domain satisfaction and global life satisfaction which was measured by the Satisfaction with Life Scale (SWLS) of Diener, Emmons, Larsen, and Griffin (1985). Using a 15-item (representing 15 domains) questionnaire to measure quality of campus life, Wu and Yao (2006a) in a different study found that domain importance did not moderate the relationship between individual domain satisfaction and global life satisfaction (measured by the SWLS). In another study, Wu and Yao (2007) conducted an experiment with 40 undergraduate students to examine the range-of-affect hypothesis, using various life areas to generate scenarios. Results of their analysis indicated that the relationship between the have-want discrepancy and satisfaction of a given domain was stronger if the domain was perceived more important (Wu \& Yao, 2007).

Citing evidence for the range-of-affect hypothesis in the life satisfaction context, 
opponents of incorporating domain importance weighting into QoL measures (Wu, 2008a, 2008b; Wu \& Yao, 2006a, 2006b, 2007) argued that domain importance weighting was redundant as importance had already been incorporated into satisfaction evaluation. Calls for abolishing incorporating domain importance weighting into QoL measures completely have been made repeatedly (Wu, 2008a, 2008b; Wu \& Yao, 2006a, 2006b, 2007).

In fact, the main argument of Locke's range-of-affect hypothesis $(1969,1976)$ spoke to the relationships between (the so-called have-want) discrepancy, importance and satisfaction within individual domains (or facets). Regarding the relationships between global satisfaction and domain satisfaction, what was postulated, based on the range-of-affect hypothesis, was that 1) it would be redundant to weight domain satisfaction with domain importance (the concept of implicit weighting) and 2) global (job) satisfaction should just be the sum of domain satisfactions (Locke, 1969, 1976). Although the rationale, based on the range-of-affect hypothesis, for the argument that importance has already been included in satisfaction evaluations was pretty straightforward, the rationale for the additive relationship between domain satisfactions and global satisfaction was less than clear. Specifically, it does not seem that the hypothesis -- global satisfaction is the sum of domain satisfactions -- can be derived from the concept of implicit weighting without assuming that the relationship between domain satisfactions is a linear and consistent one across all domains. The notion that importance has been included in satisfaction evaluations within a domain does not speak to the relationship of satisfaction between or across domains. By arguing that global satisfaction is a sum of domain satisfactions, one assumes that, among others, a consistent relationship that has the nature of perfect substitution between satisfaction across all domains (see Rojas, 2006 for a detailed discussion). However, this linear 
relationship of satisfactions across domains appeared to be more of an assumption than a hypothesis since the literature on range-of-affect hypothesis has not offered any justification for it. As a matter of fact, empirical evidence from job satisfaction literature seemed to suggest against this simple linear relationship assumption. For example, Rice, Gentile et al. (1991) showed that there was a significant curvilinear (quadratic form) relationship between global job satisfaction and domain specific satisfaction in two of the job domains studied, while the same curvilinear relationship between global job satisfaction and domain specific satisfaction was not found in other domains studied. Since the relationship between global (job) satisfaction and domain satisfaction did not appear consistent across domains, the assumption that global satisfaction was the simple sum of satisfaction across domains became arbitrary, if not questionable.

Using the concept of implicit weighting, derived from the range-of-affect hypothesis, to argue against domain importance weighting in the life satisfaction context may appear reasonable. After all, if domain importance is implicitly reflected in domain satisfaction, then it would seem redundant to weight domain satisfaction with importance repeatedly. Unfortunately, like in the job satisfaction literature, the concept of implicit weighting, in the life satisfaction context, centers on the relationship between discrepancy, importance and satisfaction within individual life domains, not between or across life domains. When it comes to the relationship between global life satisfaction and the composite of life domain satisfactions, the proposed simple linear additive relationship of the range-of-affect hypothesis (similar to the job satisfaction literature) relies on an assumption without justification. On the other hand, the relationship between global life satisfaction and the composite of domain satisfactions is the focus point of investigation of domain importance weighting. The recent study by Rojas (2006) 
detailed the limitations and implications of this assumed linear relationship (and three other alternative specifications) between global life satisfaction and domain satisfaction. As Rojas (2006) suggested, assuming a linear relationship between global life satisfaction and domain satisfaction would result in a loss of many features of the relationship and would clearly restrict our understanding of life satisfaction.

The practice of domain importance weighting is not without any conceptual ground. Although different terms have been used to describe the potential inter-person differences in perceived importance of life domains, such as "domain importance" (Campbell, Converse \& Rogers, 1976), “value priority” (Inglehart, 1978), and “psychological centrality” (Ryff \& Essex, 1992), these terms similarly reflect the concept of valence. Many researchers conceptualized the QoL measures that seemed to follow an interactive model. For example, the Ferrans and Powers' Quality of Life Index (QLI) defined QoL as “a person's sense of well-being that stems from satisfaction or dissatisfaction with the areas of life that are important to him/her" (Ferrans, 1990, p.15). Based on the definition of QoL of the Ferrans and Powers' QLI (Ferrans \& Powers, 1985), weighting domain satisfaction with importance seems conceptually justified as long as domain satisfaction and domain importance are not identical constructs. As Russell et al. (2006) pointed out, existing evidence suggested that domain satisfaction and domain importance were not identical constructs.

In short, the range-of-affect hypothesis (especially the concept of implicit weighting) focuses primarily on the relationship between global satisfaction, domain satisfaction and domain importance within individual domains. Using this hypothesis as the conceptual ground to argue against domain importance weighting, which focuses primarily on the relationship between 
global satisfaction, composite of domain satisfactions and domain importance across domains is arbitrary.

\section{Empirical Ground}

The empirical ground for the recent objection of domain importance weighting typically centered on demonstrating that domain importance did not necessarily moderate the relationship between domain satisfaction and global QoL measures (Wu \& Yao, 2006a, 2006b). The evidence that the relationship between global QoL measures and domain satisfaction scores did not seem to vary significantly across domain importance scores for individual domains led to the opposition of domain importance weighting. Unfortunately, the empirical evidence presented so far has been less than conclusive for the following reasons:

First, the moderated regression analysis in both studies by Wu \& Yao (2006a, 2006b) has only been based on a linear satisfaction by importance product/interaction term. That is, the moderated regression analysis results presented so far have assumed that, similar to the conventional multiplicative scores, the coefficient for domain satisfaction in the regression model was a linear function of domain importance rating score. More specifically, the moderated regression results presented so far have been based on the moderated regression equation that could be expressed as:

$$
y=\mathrm{A}+\mathrm{B} x_{1}+\mathrm{C} x_{2}+\mathrm{D} x_{1} x_{2}+U
$$

where $y$ is global QoL or global satisfaction, $x_{1}$ is domain satisfaction, and $x_{2}$ is domain importance.

The equation could be written as:

$$
y=\mathrm{A}+\left(\mathrm{B}+\mathrm{D} x_{2}\right) x_{1}+\mathrm{C} x_{2}+U
$$

And it could simply be considered as the following equation: 


$$
y=\mathrm{A}+\mathrm{E} x_{1}+\mathrm{C} x_{2}+U
$$

with the assumption that the coefficient for $x_{1}$ as a linear function of $x_{2}$ :

$$
\mathrm{E}=\mathrm{B}+\mathrm{D} x_{2}
$$

If a significant moderating (or interaction) effect was found in the results based on the moderated regression of the above type, it would provide the evidence that the relationship between domain satisfaction and global QoL (or satisfaction) was a linear function of domain importance rating score. On the other hand, if no significant moderating (or interaction) effect was found in the results based on the moderated regression of the above type, it would only suggest that there was no evidence to support that the relationship between domain satisfaction and global QoL (or satisfaction) was a linear function of domain importance score.

Using the moderated regression approach described above and the (arbitrarily) assumed linear scores of domain importance rating, the empirical evidence against domain importance weighting presented so far (Wu \& Yao, 2006a, 2006b) only has to do with whether or not the relationship between domain satisfaction and global QoL (or satisfaction) is a linear function of domain importance rating score and does not speak to any other, such as curvilinear or nonlinear, function of domain importance rating scores. It would be premature to suggest that the relationship between domain satisfaction and global QoL (or satisfaction) does not depend on any function of domain importance, based only on a nonsignificant (linear) moderating effect.

Second, the empirical evidence so far has been based on the argument that since domain importance did not moderate the relationship between domain satisfaction and global satisfaction for individual domains, domain satisfaction weighting would be unnecessary. This line of argument is somewhat over-generalizing. The evidence as well as argument opposing domain 
importance weighting (using moderated regression analysis) has been based on the relationship between global satisfaction or QoL, domain satisfaction, and domain importance within each individual domain (see Wu, 2008a, 2008b; Wu \& Yao, 2006a, 2006b). However, the focus of the investigation of domain importance weighting has been between and/or across domains and has been on the relationship of the composite of domain satisfactions and global satisfaction, or QoL.

It's important to note that to use satisfaction and importance of each individual domain separately to reflect (or predict) global QoL or satisfaction measures independent of other domains (though an important topic) has not been the focus of domain importance weighting. Instead, the focus of domain importance weighting, in general, is to examine the function of domain importance, using the composite of domain satisfactions, multiple domains combined at the same time. Given that the range-of-affect hypothesis and domain importance weighting each has a different focus, the empirical results, based on separate moderated regression analysis for each domain, presented so far to argue against domain importance weighting must be interpreted with caution.

To adequately examine the focus of domain importance weighting with moderated regression analysis, one should consider the three-step hierarchical regression analysis with all domains combined in one regression model suggested by Evans (1991), instead of a separate model for each domain. The analysis, similar to what was proposed by Mastekaasa (1984), would begin by estimating a regression model with global QoL as dependent variable and domain satisfaction of all the domains together as independent variables. The second step would be to add domain importance of all the domains as independent variables. The third step would be to add to the second step the interaction/product terms of domain satisfaction by importance for all domains as independent variables. The change in $R^{2}$ from stage two to stage three would 
indicate the need for the inclusion of the interaction (i.e. domain importance weighting) terms (see Evans, 1991 for detail).

To illustrate the points made above regarding moderated regression, a re-analysis of survey data from an earlier study was conducted (see Hsieh, 2003 for details). The purpose of this re-analysis was to explore beyond the typical linear function of domain importance weighting shown in previous studies (Hsieh, 2003, 2004), using the moderated regression analysis approach outlined by Evans (1991).

The global QoL was indicated by a single-item life satisfaction measure. The measure asked respondents to rate their satisfaction with life as a whole as: completely satisfied (7), somewhat satisfied (6), slightly satisfied (5), neither satisfied nor dissatisfied (4), slightly dissatisfied (3), somewhat dissatisfied (2), or completely dissatisfied (1). Respondents were also asked to rate their satisfaction in the same manner for each of the following eight domains: health, work, spare time, financial situation, neighborhood, family life, friendships, and religion. Respondents were asked to rate the importance (i.e., domain importance rating) of each of the eight discrete life domains as: not at all important (1), not too important (2), somewhat important (3), very important (4), or extremely important (5). Respondents were then asked to compare and rank among the domains with same rating response options to obtain a rank ordering of domains. In other words, this produced a hierarchy of domains from one (most important) to eight (least important) for each respondent (i.e., domain importance ranking). It was possible for domains of equal importance to be so ranked.

In order to explore the potential weighting function of domain importance, both linear and curvilinear functions of the domain importance variables were constructed. A total of seven 
different domain importance variables, corresponding to the seven types of weighting presented by Hsieh (2003), were used in the analysis. Table 1 shows the weighting types and how the importance variables were constructed.

\section{[Table 1 about here]}

Moderated regression analysis was conducted for each of type of domain importance variable (or weighting), following the three-step process outlined by Evans (1991). Since the focus was to determine the contribution of the importance by satisfaction interaction terms combined, coefficients on specific domain satisfaction, domain importance, and satisfaction by importance of specific domains were not of interest. Table 2 shows the $R^{2}$ and $R^{2}$ changes of moderated regression analysis results, based on weighting domain importance rating. As indicated earlier, the focus should be the $R^{2}$ change from step two to step three. According to the results shown in Table 2, none of the weighting type (based on domain importance rating) had a significant $R^{2}$ change from step two to step three. These results suggested that compared to a simple additive (sum of domain satisfaction scores) model, none of the weighted composite -DI, DsqrtI, or $\mathrm{DI}^{2}$-- performed better.

\section{[Table 2 about here]}

Table 3 shows the $R^{2}$ and $R^{2}$ changes of moderated regression analysis results, based on weighting domain importance ranking. As shown in Table 3, a significant $R^{2}$ change from step two to step three could be found in three of the four types of weighting. More specifically, these results suggested that except for DR, weighted composite such as DsqrtR, $\mathrm{DR}^{2}$, and Dinr would perform better, compared to a simple additive (sum of domain satisfaction scores) model.

Results from moderated regression analysis seemed to suggest that the performance of weighted composite in predicting global satisfaction had to do with how domain importance was 
measured (rating vs. ranking) as well as how the weighting was constructed (linear vs. curvilinear). It appeared that weighting via domain importance ranking with an assumed curvilinear function of domain importance, such as DsqrtR, $\mathrm{DR}^{2}$, and Dinr, could perform better than not weighting or weighting via domain importance rating in predicting global satisfaction. The results found here seem fairly consistent with the results based on correlation analysis reported previously (Hsieh, 2003, 2004).

[Table 3 about here]

\section{Discussion}

The purpose of this article is to examine the recent claims calling for abolishing domain importance weighting in QoL measures by considering the evidence both conceptually and empirically. Conceptually, the main argument against domain importance weighting was based on the range-of-affect hypothesis (Locke, 1969, 1976, 1984). More specifically, it was based on the concept of implicit weighting (Locke, 1969, 1976) extended from the range-of-affect hypothesis. The implicit weighting concept suggests that domain importance has already been reflected in domain satisfaction and therefore, weighting domain satisfaction with domain importance is redundant and unnecessary. Using the concept of implicit weighting and/or the range-of-affect hypothesis as a possible explanation for the failed performance of domain importance weighting can be of benefit to our understanding of the literature on life satisfaction and QoL. However, using the concept of implicit weighting and/or the range-of-affect hypothesis to argue for abolishing domain importance weighting would be arbitrary for the following reasons:

First, the focus of range-of-affect hypothesis is on the relationships between discrepancy, 
importance and satisfaction within individual domain. The focus of domain importance weighting, on the other hand, is on the relationships between global satisfaction or QoL and composite of satisfaction and importance across multiple domains. Demonstrating domain importance has already been reflected in domain satisfaction within individual domains does not necessarily tell us how domain satisfaction (as well as domain importance) should be combined across domains.

Second, using the concept of implicit weighting and/or the range-of-affect hypothesis to argue against domain importance weighting assumes a single construct of domain importance that not only is captured by the satisfaction evaluation process within each individual domain but also has a function that remains consistent across all domains. The plausibility of this assumption has not been examined. In fact, recent literature on consumer satisfaction showed that perceived importance of product attributes in relation to satisfaction was more than a single dimension (Smith \& Deppa, 2009). Before jumping to the conclusion that it is redundant and unnecessary to weight domain satisfaction with domain importance, the assumption of a single construct of domain importance should be evaluated.

Third, according to Locke (1976), the measurement of domain importance was by no means a simple one. It is not impossible that "the subjects asked to rate discrepancy may implicitly weight it by importance; similarly, subjects asked to rate importance may implicitly include a rating of discrepancy" (Locke, 1976, p. 1306). Therefore, it is unclear if the construct of discrepancy used to test the range-of-affect hypothesis is free from the complication of implicit weighting. Without ascertaining that the construct of discrepancy is free from implicit weighting and that implicit weighting is unique only to domain satisfactions, arguing against domain importance weighting based on redundancy is arbitrary. 
Empirically, the recent argument against domain importance weighting was based on the results of moderated regression analysis (Wu \& Yao, 2006a, 2006b). Unfortunately, the evidence used to argue that "since domain importance did not moderate the relationship between domain satisfaction and global satisfaction for individual domains, domain satisfaction weighting would be unnecessary" is over-generalizing for the following reasons:

First, results of the moderated regression analysis used to argue against domain importance weighting were based only on a linear function of domain importance rating score. A nonsignificant effect of satisfaction by importance in this case could not tell us anything about other possible functions of domain importance. It would be over-generalizing to suggest that no linear relationship means no relationship at all.

Second, results of the moderated regression analysis used to argue against domain importance weighting were based on regression models estimated for each life domain separately. The same analytical approach was used in job satisfaction literature (e.g., McFarlin et al., 1995; McFarlin \& Rice, 1992; Rice, Gentile et al., 1991) and by Marsh (1986) to study the relationships between global self-esteem and specific facets of self-concept and their importance. The separate analysis done for each individual life domain offered evidence on the relationship between global life satisfaction (or QoL), domain satisfaction and importance for each specific life domain, not multiple life domains combined. It is not the general focus of the investigation of domain importance weighting to study how satisfaction with a single life domain relates to global life satisfaction or QoL. Instead, the general focus of the investigation of domain importance weighting is to study how satisfaction with multiple life domains combined relates to global life satisfaction or QoL. Using empirical evidence with a focus on each single life domain, 
rather than multiple life domains combined, to argue against domain importance weighting would be over-generalizing.

Third, as shown in the results of moderated regression analysis presented in this study, the performance of weighted domain satisfaction composites could vary significantly, depending on the choice of weighting method and the domain importance measure. These findings are quite consistent with the results of correlation analysis shown in previous studies (Hsieh, 2003, 2004). That is, whether or not weighted domain satisfaction composites would perform significantly better (than unweighted composite) in predicting (or correlating with) global satisfaction or QoL measures is a result of the interplay of a multitude of factors, including, among others, the specific global QoL measure(s) chosen (Russell \& Hubley, 2005; Russell et al., 2006), the way domain importance measured, the domains of life (how exhaustive) included, and the type of weighting approach selected (Hsieh, 2003, 2004). Arguing against domain importance weighting, using evidence based on a limited choice of global satisfaction or QoL measures, measurements of domain importance, scoring systems of domain importance, and weighting approaches, is over-generalizing.

In sum, using the range-of-affect hypothesis as a possible explanation of the poor performance of weighted satisfaction composite in predicting or correlating with global satisfaction or QoL measures can be beneficial to our understanding of the life satisfaction literature. However, given the conceptual focus and the empirical approach of the range-of-affect hypothesis presented so for in the life satisfaction context, using the range-of-affect hypothesis to argue against domain importance weighting raised more questions than answers. Calling for abolishing domain importance weighting in QoL measures, based on the evidence of range-ofaffect hypothesis, is premature. 


\section{References}

Campbell, A., Converse, P. E., and W. L. Rogers: 1976, The Quality of American Life: Perceptions, Evaluations, and Satisfactions (Russel Sage, New York).

Diener, E., Emmons, R.A., Larsen, R.J. and Griffin, S.: 1985, 'The satisfaction with life scale', Journal of Personality Assessment 49, pp. 71-74.

Evans, M.G.: 1991, 'The problem of analyzing multiplicative composites: Interactions revisited', American Psychologist 46, pp. 6-15.

Ferrans, C. E.: 1990, 'Development of a quality of life index for patients with cancer', Oncology Nursing Forum 17(3), pp. 15-19.

Ferrans, C. E. and M. J. Powers: 1985, 'Quality of life index: Development and psychometric properties', Advances in Nursing Science 8(1), pp. 15-24.

Hsieh, C. M.: 2003, 'Counting importance: The case of life satisfaction and relative domain importance', Social Indicators Research 61, pp. 227-240.

Hsieh, C. M.: 2004, 'To weight or not to weight: The role of domain importance in quality of life measurement', Social Indicators Research 68, pp. 163-174.

Inglehart, R.: 1978, 'Value priorities, life satisfaction, and political dissatisfaction among western publics', Comparative Studies in Sociology 1, pp. 173-202.

Locke, E.A.: 1969, 'What is job satisfaction?', Organizational Behavior and Human Performance 4, 309-336.

Locke, E. A.: 1976, ‘The nature and causes of job satisfaction', pp. 1297-1349 in Handbook of Industrial and Organizational Psychology, edited by M. D. Dunnette (Rand McNally, Chicago). 
Locke, E. A.: 1984, ‘Job satisfaction', pp. 93-117 in Social Psychology and Organizational Behavior, edited by M. Gruneberg and T. Wall (Wiley, London).

Marsh, H.W.: 1986, 'Global self-esteem: Its relation to specific facets of self-concept and their importance', Journal of Personality and Social Psychology 51, pp. 1224-1236.

Mastekaasa, A.: 1984, 'Multiplicative and additive models of job and life satisfaction', Social Indicators Research 14, pp. 141-163.

McFarlin, D.B., E.A. Coster, R.W. Rice and T. Coopper-Alison: 1995, 'Facet importance and job satisfaction: Another look at the range of affect hypothesis', Basic and Applied Social Psychology 16, 489-502.

McFarlin, D.B. and R.W. Rice: 1992, 'The role of facet importance as a moderator in job satisfaction processes', Journal of Organizational Behavior 13, pp. 41-54.

Rice, R.W., D.A. Gentile and D.B. McFarlin: 1991, 'Facet importance and job satisfaction', Journal of Applied Psychology 76, pp. 31-39.

Rice, R.W., K. Markus, R.P. Moyer and D.B. McFarlin: 1991, 'Facet importance and job satisfaction: Two experimental tests of Locke's range of affect hypothesis', Journal of Applied Social Psychology 21, pp. 1977-1987.

Rojas, M.: 2006, 'Life satisfaction and satisfaction in domains of life: Is it a simple relationship?' Journal of Happiness Studies 7, pp. 467-497.

Russell, L. B. and A. M. Hubley: 2005, 'Importance ratings and weighting: Old concerns and new perspectives', International Journal of Testing 5, pp. 105-130. 
Russell, L. B., A. M. Hubley, A. Palepu and B. D. Zumbo: 2006, 'Does weighting capture what's important? Revisiting subjective importance weighting with a quality of life measure', Social Indicators Research 75, pp. 146-167.

Ryff, C. D. and M. J. Essex: 1992, 'The interpretation of life experience and well-being: The sample case of relocation', Psychology and Aging 7, pp. 507-517.

Skalli, A., I. Theodossioub and E Vasileioua: 2008, 'Jobs as Lancaster goods: Facets of job satisfaction and overall job satisfaction', Journal of Socio-Economics 37, pp. 1906-1920.

Smith, R. and B. Deppa: 2009, 'Two dimensions of attribute importance', Journal of Consumer Marketing 26, pp. 28-38.

Trauer, T. and A. Mackinnon: 2001, 'Why are we weighting? The role of importance ratings in quality of life measurement', Quality of Life Research, 10, pp. 579-585.

Wu, C. H.: 2008a, 'Examining the appropriateness of importance weighting on satisfaction score from range-of-affect hypothesis: Hierarchical linear modeling for within-subject data', Social Indicators Research 86, pp. 101-111.

Wu, C. H.: 2008b, 'Can we weight satisfaction score with importance ranks across life domains?', Social Indicators Research 86, pp. 468-480.

Wu, C. H. and G. Yao: 2006a, 'Do we need to weight item satisfaction by item importance? A perspective from Locke's range-of-affect hypothesis', Social Indicators Research 79, pp. $485-502$.

Wu, C. H. and G. Yao: 2006b, 'Do we need to weight satisfaction scores with importance ratings in measuring quality of life?', Social Indicators Research 78, pp. 305-326. 
Wu, C. H. and G. Yao: 2007, 'Importance has been considered in satisfaction evaluation: An experimental examination of Locke's range-of-affect hypothesis', Social Indicators Research 81, pp. 521-541. 
Table 1

Correspondence of Weighting Type, Domain Importance Variable and Weighted Composite Domain Importance Variable

Weighted Composite ${ }^{\mathrm{a}}$

Weighting Type

Importance rating (I)

DI

$\mathrm{I}_{i} / \sum \mathrm{I}_{i}$

$\sum\left(\mathrm{S}_{i} \times \mathrm{I}_{i}\right) / \sum \mathrm{I}_{i}$

DsqrtI

$\operatorname{sqrt}\left(\mathrm{I}_{i}\right) / \sum \operatorname{sqrt}\left(\mathrm{I}_{i}\right)$

$\sum\left(\mathrm{S}_{i} \times \operatorname{sqrt}\left(\mathrm{I}_{i}\right)\right) / \sum \operatorname{sqrt}\left(\mathrm{I}_{i}\right)$

$\mathrm{DI}^{2}$

$\mathrm{I}_{i}^{2} / \sum \mathrm{I}_{i}^{2}$

$\sum\left(\mathrm{S}_{i} \times \mathrm{I}_{i}^{2}\right) / \sum \mathrm{I}_{i}^{2}$

Importance ranking $(\mathrm{R})^{\mathrm{b}}$

DR

$\mathrm{R}_{i} / \sum \mathrm{R}_{i}$

$\sum\left(\mathrm{S}_{i} \times \mathrm{R}_{i}\right) / \sum \mathrm{R}_{i}$

DsqrtR

$\operatorname{sqrt}\left(\mathrm{R}_{i}\right) / \sum \operatorname{sqrt}\left(\mathrm{R}_{i}\right)$

$\sum\left(\mathrm{S}_{i} \times \operatorname{sqrt}\left(\mathrm{R}_{i}\right)\right) / \sum \operatorname{sqrt}\left(\mathrm{R}_{i}\right)$

$\mathrm{DR}^{2}$

$\mathrm{R}_{i}^{2} / \sum \mathrm{R}_{i}^{2}$

$\sum\left(\mathrm{S}_{i} \times \mathrm{R}_{i}{ }^{2}\right) / \sum \mathrm{R}_{i}{ }^{2}$

Dinr $^{\mathrm{c}}$

$\left(1 / \mathrm{r}_{i}\right) / \sum\left(1 / \mathrm{r}_{i}\right)$

$\sum\left(\mathrm{S}_{i} / \mathrm{r}_{i}\right) / \sum\left(1 / \mathrm{r}_{i}\right)$

Notes. ${ }^{\text {a }} \mathrm{S} i$ is the satisfaction rating in domain $i$

${ }^{\mathrm{b}} \mathrm{R}$ is the reverse value of domain ranking

${ }^{\mathrm{c}} \mathrm{r}$ is the value of domain ranking

See Hsieh (2003) for details. 
Table 2

Results of Moderated Regression Analysis with Importance Rating Predicting Global Satisfaction

\begin{tabular}{lccc}
\hline & Step 1 & Step 2 & Step 3 \\
Weighting Type & Satisfaction $R^{2}$ & Importance $\Delta R^{2}$ & Satisfaction x Importance $\Delta R^{2}$ \\
\hline DI & $.237 * *$ & .086 & .126 \\
DsqrtI & $.237^{* * *}$ & .087 & .125 \\
& & & \\
$\mathrm{DI}^{2}$ & $.237 * *$ & .085 & .127 \\
\hline$* * p<.01$ & &
\end{tabular}


Table 3

Results of Moderated Regression Analysis with Importance Ranking Predicting Global Satisfaction

\begin{tabular}{|c|c|c|c|}
\hline Weighting Type & $\begin{array}{c}\text { Step } 1 \\
\text { Satisfaction } R^{2}\end{array}$ & $\begin{array}{c}\text { Step } 2 \\
\text { Importance } \Delta R^{2}\end{array}$ & $\begin{array}{c}\text { Step } 3 \\
\text { Satisfaction } \mathrm{x} \text { Importance } \Delta R^{2}\end{array}$ \\
\hline DR & $.237 * *$ & .011 & .129 \\
\hline DsqrtR & $.237 * *$ & .047 & $.165^{*}$ \\
\hline $\mathrm{DR}^{2}$ & $.237 * *$ & .044 & $.181 * *$ \\
\hline Dinr & $.237 * *$ & .068 & $.169 *$ \\
\hline
\end{tabular}


Chang-ming Hsieh

Jane Addams College of Social Work

University of Illinois at Chicago

1040 West Harrison Street (M/C 309)

Chicago, IL 60607-7134

Email: chsieh@uic.edu 